\title{
The range of multiple sclerosis associated with neurofibromatosis type 1
}

\author{
P Perini, P Gallo
}

\begin{abstract}
Multiple sclerosis associated with neurofibromatosis type 1 (NF1) is a very rare event. Seven patients with multiple sclerosis and NF1 are described in the literature, and all were reported to have the primary progressive form of multiple sclerosis. Three new patients with NF1 that developed multiple sclerosis are described and it is shown that the range of multiple sclerosis associated with NF1 includes the relapsing forms of the disease. The risk of having both NF1 and multiple sclerosis in north east Italy is higher than would be expected based on the prevalence rates of the two diseases. (F Neurol Neurosurg Psychiatry 2001;71:679-681)
\end{abstract}

Keywords: multiple sclerosis; neurofibromatosis

Neurofibromatosis type 1 (NF1) is an autosomal dominant disorder the gene of which is located on chromosome 17q11.2. ${ }^{1}$ In white people, it has a prevalence ranging from $1 / 2190$ to $1 / 7800$, and a spontaneous mutation rate of about $50 \% .^{2-5}$ The neurological manifestation of NF1 consists of mild intellectual impairment, optic nerve and parenchymal gliomas, epilepsy, and macrocephaly. ${ }^{67}$ The rare and exclusive association of NF1 with the primary progressive form of multiple sclerosis has been described ${ }^{8-10}$; this association is particularly interesting because the gene for oligodendrocyte myelin glycoprotein (OMgp) is embedded within intron $27 \mathrm{~b}$ of the NF1 gene $\mathrm{A},{ }^{11}$ and a single mutation in this gene, which resulted in an amino acid change from glycine to aspartic acid at codon 21 , was found in $16.6 \%$ of patients with multiple sclerosis. ${ }^{12}$ This mutation, however, was found to be neither sufficient nor necessary for the development of the primary progressive form of the disease. ${ }^{10}$ Indeed, only seven patients with NF1 and primary progressive multiple sclerosis have been described in the literature to date. Here, we report on three more patients with NF1 who developed multiple sclerosis, one with primary progressive, one with secondary progressive, and one with the relapsing-remitting form of the disease

\section{Case 1}

and in revised form

20 April 2001

Accepted 25 April 2001 of café au lait spots, axillary freckling, and multiple cutaneous neurofibromas. He had no family history of NF-1, but the search for a mutation in the NF1 gene was positive. In 1998, at 19 years of age, he presented with internuclear ophthalmoplegia, diplopia, and nystagmus on horizontal gaze. There was a mild disequilibrium, and neurological examination showed hyperreflexia, bilateral Babinski's sign, and mild distal hypopallestesia. Brain MRI showed multiple areas of altered signal in the white matter of both cerebral hemispheres, predominantly in the centrum semiovale and periventricular regions, and the corpus callosum (two lesions); some of them were enhanced by gadolinium-EDTA. Examination of the CSF disclosed intrathecal IgG synthesis (increased IgG indices and IgG oligoclonal bands), and mild pleiocytosis (12 lymphocytes/ $\mu \mathrm{l})$. Visual evoked potentials (VEPs) and somatosensory evoked potentials (SSEPs) gave abnormal responses. Detailed biochemical, immunological, and haematological screening showed variables within the normal range. He had clear benefit from high dose steroid therapy (methylprednisolone (1 $\mathrm{g}$ /day for 6 days)), with the disappearance of all clinical symptoms and signs. One year later, he developed left side paraesthesias. Brain MRI showed new gadolinium enhancing lesions and an increased T2 lesion load. Again, he was treated with high dose steroids (1 g/day for 6 days), and the symptoms almost disappeared. The diagnosis of clinically definite relapsingremitting multiple sclerosis was formulated. After high dose steroid therapy, his score on the expanded disability status scale (EDSS) examination was 1 point. One year later, the patient was asymptomatic, and his EDSS score was unchanged.

\section{Case 2}

A 44 year old woman was diagnosed with NF1 when she was 29 years old on the basis of café au lait patches, axillary freckling, and multiple cutaneous neurofibromas. There was no family history of NF1. At the age of 30, she developed bilateral glove-like paraesthesias that spontaneously disappeared within 2 months. Three years later she complained of painful tingling in the hands, legs, and feet, and was studied further. The search for NF1 gene mutation was positive. Brain and spinal cord MRI showed 
multiple T2 areas of altered signals, one of which was in the cervical spinal cord. Examination of CSF showed IgG oligoclonal bands, increased IgG indexes, and eight lymphocytes/ $\mu 1$. Brain stem auditory evoked responses (BAERs), VEPs, and SSEPs were abnormal. Multiple sclerosis was diagnosed, and high dose steroid therapy proved beneficial. In the next 4 years she had two more relapses, which were treated with high dose steroids and she experienced almost complete recovery. In 1993 she had a new relapse, and the disease entered a slowly progressive phase. She developed weakness and stiffness of the legs, overactive bladder, and painful paraesthesias at the feet. Her EDSS score was 4. High dose steroids did not bring about an obvious improvement. She came to us when she was 44 years old, with an EDSS score of 4.5. Brain MRI showed a significant increase in T2 lesion load, but no new gadolinium enhancing lesions. Her VEPs, BAERs, and SSEPs were abnormal, and secondary progressive multiple sclerosis was diagnosed.

\section{Case 3}

A 50 year old white man with a positive family history for NF1 developed clinically evident NF1 at the age of 16 . When he came to us he had a history of slowly progressive weakness and stiffness of his legs. Four years earlier he had developed sphincter dyssynergia and bilateral paraesthesias at the feet. During the past year he also became progressively ataxic and developed a mild cerebellar tremor in his left arm. His EDSS score was 5.5. Brain MRI showed multiple predominantly periventricular T2 lesions in both cerebral hemispheres; some lesions were evident in the corpus callosum, which was notably atrophic; only one lesion was enhanced by gadolinium-EDTA. Examination of CSF showed normal cell count, increased IgG index, and the presence of IgG oligoclonal bands. His VEPs, BAERs, and SSEPs were abnormal. Detailed immunological and haematological examination, as well as tests for leukodystrophy and autoantibodies were negative. He had only mild and transient benefit from high dose steroid therapy; primary-progressive multiple sclerosis was diagnosed.

\section{Discussion}

The association of multiple sclerosis with NF1 is a very rare event. Indeed, only seven patients with NF1 and the primary progressive form of multiple sclerosis are described in the literature. ${ }^{3-10}$ In this report we describe three more patients with NF1 who subsequently developed clinically definite multiple sclerosis, two of whom had the relapsing form. The diagnosis of multiple sclerosis was confirmed by typical MRI findings, the presence of IgG oligoclonal bands, and increased IgG indexes in the CSF (in two cases there was also a mild pleiocytosis), and the abnormal responses to evoked potentials. These cases clearly show that all the clinical forms of multiple sclerosis can be found in patients with NF1. All three patients described were originally from two regions of north east Italy-Veneto and Friuli-Venezia Giulia, with a joint population in the 1991 census of 5578500 inhabitants. A recent epidemiological survey in this area estimated a multiple sclerosis prevalence of about 80/100 000 (Gallo P et al, unpublished data). There is no recent epidemiological survey for NF1 in our region, but a previous study in patients from Veneto and Friuli-Venezia Giulia who were referred for genetic counselling showed a prevalence of $1 / 6711(15 / 100000) \cdot{ }^{13}$ Although the prevalence of NF1 among people living in north east Italy is probably underestimated, based on the prevalence of 20:100 000 found in other white populations, we would expect to have only $0.016 / 100000$ patients with both diseases in our region - that is, less than 0.9 patients in 5.5 million inhabitants (assuming the prevalence of $\mathrm{NF} 1=15 / 100000$, only $0.012 / 100000$ patients with both diseases are expected-0.66 patients/5.5 million inhabitants). Because the three patients described here were identified among 1000 patients with multiple sclerosis examined at the Centre for Multiple Sclerosis in Padova, the prevalence of the association of multiple sclerosis with NF1 can be calculated as $\mathrm{P}(\mathrm{SMnNF} 1)=(80 / 100000) \times$ $(3 / 1000) \times 100000)=0.24 / 100000-15$ times higher than expected $(\mathrm{P}(\mathrm{SM}) \times \mathrm{P}(\mathrm{NF} 1)=(80 /$ $100000) \times(20 / 100000)=0.016 / 100000 ;$ exact Poisson test: $\mathrm{p}=0.001)$. These figures clearly indicate an association between multiple sclerosis with NF1.

This association is particularly interesting because, as mentioned above, the gene for OMgp, a membrane glycoprotein which might be one of the possible target antigens of the autoimmune attack in demyelinating diseases, is embedded within intron $27 \mathrm{~b}$ of the NF1 gene, located on chromosome $17 \mathrm{q} 11.2,{ }^{11}$ and a single mutation in the OMgp gene may occur in patients with multiple sclerosis. ${ }^{12}$ Moreover, patients with NF1 with gross deletions may lose at least 11 functional genes, including OMgp. ${ }^{14}$ However, a recent study has shown that the primary progressive form of multiple sclerosis can occur in patients with NF1 without concurrent mutation in the MOgp genethat is, the glycine to aspartic acid polymorphic alteration at codon 21 is neither sufficient nor necessary for the development of a progressive inflammatory white matter disease. ${ }^{10}$

Besides the genetic hypothesis, it must also be considered that NF1 is mainly a proliferative disease involving Schwann cells. In patients with NF1, it might be possible that an abnormal exposure to peripheral myelin antigens activates an autoimmune response to the same antigens expressed in CNS myelin. Finally, almost all the clinical features of NF1 indicate that the NF1 gene is mainly a "suppressor gene", which exerts its action on many cell types and organs (Schwann cells, melanocytes, fibroblasts, etc $)^{15}$; if it also suppresses the cells of the immune system, then a loss of this activity might favour an autoimmune response to CNS myelin in susceptible patients. 
We thank Ms Patricia Segato for excellent revision of the language, Dr Mario Ermani for the statistical analysis, and Professor G Biasi for useful discussion.

1 Shen MH, Harper PS, Upadhyaya M. Molecular genetics of neurofibromatosis type 1 (NF-1). 7 Med Genet 1996;33:1-17.

2 Huson SM, Harper PS, Compston DAS. Von Reckiinghausen neurofibromatosis: a clinical and population study in south east Wales. Brain 1988;111:1355-81.

3 Huson SM, Compston DAS, Clark P, et al. A genetic study of von Recklinghausen disease in south Wales. I Prevalence, fitness, mutation rate, and effect of parental transmission on severity. F Med Genet 1989;26:704-11.

4 Samuelsson B, Samuelsson S. Neurofibromatosis in Gothenburg, Sweden. I. Background, study design, and epidemiology. Neurofibromatosis 1989;2:6-22.

5 Friedman JM. Epidemiology of neurofibromatosis type I. Am 7 Med Genet 1999;89:1-6.

6 Ferner RE, Hughes RAC, Weinman J. Intellectual impairment in neurofibromatosis 1. F Neurol Sci 1996;138:12533.
7 Creange A, Zeller J, Rostaing-Rigattieri S, et al. Neurologial complications of neurofibromatosis in adulthood. Brain 999;122:473-81.

8 Ferner RE, Hughes RAC, Johnson MR. Neurofibromatosis 1 and multiple sclerosis. F Neurol Neurosurg Psychiatry 1995;58:582-5

9 Masson C, Colombani J-M. Neurofibromatose 1 et sclérose en plaques. Rev Neurol 1997;153:684-6.

10 Johnson MR, Ferner RE, Bobrow M, et al. Detailed analysis of the oligodendrocyte myelin glycoprotein gene in four patients with neurofibromatosis 1 and primary progressive multiple sclerosis. F Neurol Neurosurg Psychiatry 2000;68: 643-6.

$11 \mathrm{Li} \mathrm{Y}$, O'Connel $\mathrm{P}$, Breindenbach $\mathrm{HH}$, et al. Genomic organization of the neurofibromatosis 1 gene (NF1). Genomics 1995;25:9-18.

12 Hinks LJ, Price SE, Mason CR, et al. Single strand conformation analysis of two genes contained in the first intron of the neurofibromatosis type 1 gene in patients with multiple sclerosis. Neuropathol Appl Neurobiol 1995;21:201-7.

13 Clementi M, Barbujani G, Turolla L, et al. Neurofibromatosis-1: a maximum likelihood estimation of Neurofibromatosis-1: a maximum likelihood

14 Jenne DE, Tinschert S, Stegmenn E, et al. A common set of Jenne DE, Tinschert S, Stegmenn E, et al. A common set of
at least 11 functional genes is lost in the majority of NF1 patients with gross deletions. Genomics 2000;66:93-7.

15 Viskochil D. Neurofibromatosis 1. Am $\mathcal{f}$ Med Genet 1999;89:v-vii.

\section{7th European Forum on Quality Improvement in Health Care}

\section{1-23 March 2002 \\ Edinburgh, Scotland}

We are delighted to announce this forthcoming conference in Edinburgh. Authors are invited to submit papers (call for papers closes on Friday 5 October 2001) and delegate enquiries are welcome.

The themes of the Forum are:

- Leadership, culture change, and change management

- Achieving radical improvement by redesigning care

- Health policy for lasting improvement in health care systems

- Patient safety

- Measurement for improvement, learning, and accountability

- Partnership with patients

- Professional quality: the foundation for improvement

- Continuous improvement in education and training

- People and improvement.

Presented to you by the BMJ Publishing Group (London, UK) and Institute for Healthcare Improvement (Boston, USA). For more information contact: quality@bma.org.uk or look at the website www.quality.bmjpg.com. Tel: +44 (0)20 7383 6409; fax: +44 (0)20 73736869 . 